\title{
Distal Femoral Varus Osteotomy for Lateral Compartment Osteoarthritis in the Valgus Knee. A Systematic Review of the Literature
}

\author{
Adnan Saithna ${ }^{*}, 1$, Rik Kundra ${ }^{2}$, Chetan S. Modi ${ }^{1}$, Alan Getgood ${ }^{1}$ and Tim Spalding ${ }^{1}$ \\ ${ }^{1}$ University Hospitals Coventry and Warwickshire NHS Trust, Clifford Bridge Road, Walsgrave, Coventry, CV2 2DX, \\ $U K$ \\ ${ }^{2}$ Walsall Healthcare NHS Trust, Moat Road, Walsall, West Midlands, WS2 9PS, UK
}

\begin{abstract}
The primary objectives of this systematic review were to define the indications, functional outcomes, survivorship and complications associated with distal femoral varus osteotomy (DFVO).

Cumulative survival with arthroplasty as the endpoint ranged from 64 to $82 \%$ at 10 years, and $45 \%$ at 15 years. The mean pre-operative HSS score ranged from 46 to 65 and this improved at latest follow up to means of between 72 and 88 . Pooled results show an overall complication rate of $5.8 \%(5 / 86)$ for unanticipated re-operation due to a complication.

Poor reporting of included studies and considerable heterogeneity between them precluded any statistical analysis. Further study is required to determine the precise indications for DFVO, optimum surgical technique, implant of choice and postoperative rehabilitation regimen as all of these factors may significantly affect the complication profile and outcomes of this procedure.

DFVO is technically demanding and requires a significant period of rehabilitation for the patient. However, long-term survivorship and good function have been demonstrated and it remains a potential option for valgus osteoarthritis in carefully selected patients.
\end{abstract}

Keywords: Distal femoral varus osteotomy, outcome measures, complications, arthroplasty, valgus osteoarthritis.

\section{INTRODUCTION}

Lateral compartment gonarthrosis in the young patient represents a challenge for the orthopaedic surgeon. Although Total Knee Arthroplasty (TKA) reliably offers good symptomatic relief and long-term implant survivorship in elderly patients, the 3 - to 5- fold higher risk of revision surgery in patients younger than 55 years of age is a cause for concern [1].

Although good results from arthroplasty are achievable, there remains a logical reluctance to avoid joint replacement in young patients. An alternative option is realignment osteotomy. This joint preserving procedure aims to reduce pain and the risk of progression of degenerative change by correcting deformity, offloading the affected compartment and potentially allowing a return to heavy functional loading that could jeopardise the survival of a joint replacement [2]. Good to excellent outcome of osteotomy for correction of varus deformity of the knee is reported in up to $92 \%$ of patients at 5 years [3]. Valgus deformity associated with lateral gonarthrosis is much less common and as a result there are fewer studies reporting outcome of osteotomy in this population.

Long-term case series report 10-year survivorship of 64$82 \%$ for closing wedge distal femoral varus osteotomy

*Address correspondence to this author at the University Hospitals Coventry and Warwickshire NHS Trust, Clifford Bridge Road, Walsgrave, Coventry, CV2 2DX, UK; Tel: 02476 964000; Fax: 02476 966456;

E-mail: adnan.saithna@gmail.com
(DFVO) using conversion to TKA as an endpoint $[4,5]$. However, the precise role of DFVO in the management of lateral compartment gonarthrosis remains undefined, particularly with respect to the amount of wear that indicates or contraindicates the procedure in early and late arthritis. In view of the absence of controlled trials and comparative studies, the purpose of this study was to conduct a systematic review to define the indications, functional outcomes, survivorship and complications associated with DFVO.

\section{METHODS}

Three independent reviewers (A.S., R.K., C.S.M.) separately applied the search strategy to PubMed, Medline and Cochrane databases. The search terms included distal, femur, femoral, varus, and osteotomy. Potentially eligible papers were manually reviewed and were discussed among the authors. References of retrieved articles were searched for further relevant studies. A decision was made regarding inclusion after application of the eligibility criteria.

Studies were considered for inclusion if they were published in the English language and the main focus of the article was distal femoral varus osteotomy for lateral compartment osteoarthritis of the knee.

A total of 13 potentially relevant studies were identified [5-16]. Reasons for subsequent exclusion were articles that concentrated solely on surgical technique, the use of external fixation as the predominant mode of fixation [11], inclusion of patients with diagnoses including inflammatory arthropathy and deformity secondary to trauma $[9,10]$, the use of multiple surgical techniques in a small case series [7], 
and the use of plaster cast rather than internal fixation [14]. Two studies [5,6] reported on the same group of patients and therefore only the study reporting longest follow up was included [5]. The search of the Cochrane library retrieved one potentially relevant review but it was found that the authors had identified no studies reporting DFVO and it was therefore also excluded [13]. A total of 6 studies were therefore included for review $[4,5,8,12,15,16]$ (see Fig. 1). These were appraised by two independent reviewers (A.S. and C.S.M.) with respect to a validated quality assessment scale for retrospective clinical studies developed by Rangel, et al. [17]. The tool allows identification of the most rigorous evidence by characterising 16 baseline criteria that define thorough and accurate reporting of non-controlled studies. If there was any disagreement between the authors in assigning a score to each paper appraised, a third independent reviewer (R.K.) made the final decision.

\section{RESULTS}

The reporting criteria met by included studies with respect to the critical appraisal tool proposed by Rangel, et al. [17] are presented in Table 1. Relevant data extracted from studies including the functional, clinical and radiological outcome are presented in Table $\mathbf{2}$ and complications are presented in Table $\mathbf{3}$.

Backstein, et al. [4], reported a case series of closing wedge DFVO in 38 patients with a mean follow up of 123 months. The closing wedge technique used in this study was originally described by McDermott, et al. [6]. Following transverse osteotomy, a 90 degree offset dynamic compression blade plate was fixed to the shaft of the femur after inserting the blade parallel to the articular surface. The intention was to correct the anatomical tibiofemoral angle to 0 degrees. Patients were allowed to fully weight bear at 6-8 weeks post-operatively [5]. The main outcomes reported were an improvement in the mean knee society objective score (pre-operative 18, range $0-74$, post-operative 87.2, range 50-100) and function scores (pre-operative 54, range $0-100$, post-operative 85.6 , range $40-100$ ) and 10 and 15 year survival figures of $82 \%(95 \%$ CI $75 \%-89 \%)$ and $45 \%$ (33\%-57\%) respectively (with conversion to TKA as the endpoint). Overall this series satisfied 11/16 of the criteria proposed by Rangel et al. [17]. Eligibility criteria for the study and indications for surgery were not defined. Specifically, it was not stated whether the authors excluded patients with degenerative change in other compartments. No complications were reported and although it is possible that none occurred this is not stated.

Edgerton, et al. [8] reported a series of 23 patients who underwent DFVO for painful genu valgum secondary to lateral osteoarthritis. Patients with radiologically mild/moderate and severe changes in the lateral compartment were included. Some patients with severe changes in the medial and patellofemoral joints were also included. The same surgical technique was not used throughout the study and detail regarding pre-operative planning and the degree of deformity correction intended were not clearly reported. Complications occurred in $63 \%$ of cases and included nonunion and loss of correction. The authors attributed this high rate of complications to the use of staple fixation, which they have subsequently abandoned. However, details of postoperative rehabilitation were not reported and may also have influenced the rate of loss of fixation. The mean pre-op HSS score was 58 (27 to 82$)$ and mean post op score was 78 (40-

Table 1. Adequacy of Reporting of Studies Based on Criteria Proposed by Rangel et al.

\begin{tabular}{|c|c|c|c|c|c|c|}
\hline Criteria of Rangel et al. & Edgerton & Finkelstein & Stahelin & Wang & Backstein & Das \\
\hline $\begin{array}{l}\text { Description of participating surgeons/institutions: } \\
\text { Can number of centres be determined? } \\
\text { Can practice type be determined? } \\
\text { Can number of surgeons be determined? } \\
\text { Can author's prior experience with procedure be determined? } \\
\text { Is timeline of when cases performed documented? } \\
\text { Description and definition of cases: } \\
\text { Was population from which cases selected described? } \\
\text { Are diagnostic criteria clearly documented? } \\
\text { Are eligibility criteria clearly documented? } \\
\text { Description of the intervention: } \\
\text { Is surgical technique adequately described? } \\
\text { Any attempt to standardise operative technique mentioned? } \\
\text { Any attempt to standardise peri-operative care mentioned? } \\
\text { Analysis of outcome data: } \\
\text { Is mean and range of demographic data reported? } \\
\text { Are outcomes presented with appropriate measures of variability? } \\
\text { Are diagnostic methods for defining outcome described? } \\
\text { Do authors address whether there is missing data? } \\
\text { Is number and nature of complications addressed? }\end{array}$ & $\begin{array}{c}\text { No } \\
\checkmark \\
\text { No } \\
\text { No } \\
\text { No } \\
\text { No } \\
\checkmark \\
\checkmark \\
\checkmark \\
\checkmark \\
\checkmark\end{array}$ & $\begin{array}{l}\text { No } \\
\text { No } \\
\text { No } \\
\checkmark \\
\checkmark \\
\checkmark \\
\checkmark \\
\checkmark \\
\checkmark \\
\checkmark \\
\checkmark\end{array}$ & $\begin{array}{c}\text { No } \\
\checkmark \\
\text { No } \\
\checkmark \\
\checkmark \\
\checkmark \\
\checkmark \\
\checkmark \\
\checkmark \\
\checkmark \\
\checkmark\end{array}$ & $\begin{array}{c}\text { No } \\
\checkmark \\
\text { No } \\
\checkmark \\
\checkmark \\
\checkmark \\
\checkmark \\
\checkmark \\
\checkmark \\
\text { No } \\
\checkmark\end{array}$ & $\begin{array}{l}\text { No } \\
\text { No } \\
\text { No } \\
\checkmark \\
\checkmark \\
\checkmark \\
\checkmark \\
\checkmark \\
\checkmark \\
\checkmark \\
\text { No }\end{array}$ & $\begin{array}{c}\checkmark \\
\checkmark \\
\text { No } \\
\text { No } \\
\checkmark \\
\text { No } \\
\checkmark \\
\checkmark \\
\checkmark \\
\checkmark \\
\checkmark \\
\checkmark \\
\text { No } \\
\checkmark \\
\checkmark \\
\checkmark\end{array}$ \\
\hline Total number of criteria satisfied & $9 / 16$ & $11 / 16$ & $12 / 16$ & $11 / 16$ & $11 / 16$ & $12 / 16$ \\
\hline
\end{tabular}


Table 2. Data Extracted from Included Studies

\begin{tabular}{|c|c|c|c|c|c|c|}
\hline & Edgerton & Finkelstein & Stahelin & Wang & Backstein & Das \\
\hline Year of publication & 1993 & 1996 & 2000 & 2005 & 2007 & 2008 \\
\hline Study design & Case Series & Case Series & Case Series & Case Series & Case Series & Case Series \\
\hline $\begin{array}{l}\text { Number of patients } \\
\text { (number of knees) }\end{array}$ & $23(24)$ & $20(21)$ & $19(21)$ & $30(30)$ & $38(40)$ & $12(12)$ \\
\hline Gender ratio M:F & $5: 18$ & $14: 6$ & $10: 9$ & $2: 28$ & $10: 28$ & $5: 7$ \\
\hline $\begin{array}{l}\text { Mean age of patients } \\
\text { in years (range) }\end{array}$ & $55(37-70)$ & $56(27-77)$ & $57(39-71)$ & $53(31-64)$ & $44.1(20-67)$ & $55(46-71)$ \\
\hline $\begin{array}{c}\text { Mean follow up } \\
\text { (months unless } \\
\text { stated) }\end{array}$ & $8.3(5-11)$ years & 133(97-240) & $5(2-12)$ years & $99(61-169)$ & $123(39-245)$ & $74(51-89)$ \\
\hline $\begin{array}{l}\text { Wear in other } \\
\text { compartments } \\
\text { allowed }\end{array}$ & yes & yes & no & yes & not stated & Yes \\
\hline $\begin{array}{l}\text { Mean pre-op valgus } \\
\text { deformity (degrees) }\end{array}$ & $18(10-27)$ & not reported & $12(10-16)$ & $18.2(12-27)$ & $11.6(4-15)$ & $16(10-21)$ \\
\hline $\begin{array}{l}\text { Intended correction } \\
\text { (tibiofemoral angle) }\end{array}$ & not stated & 0 degrees & $1-3$ varus & 0 degrees & O degrees & 3 valgus \\
\hline $\begin{array}{c}\text { Mean post-op } \\
\text { angulation (degrees) }\end{array}$ & $\begin{array}{c}1 \text { valgus }(18 \text { varus- } \\
16 \text { valgus }\end{array}$ & not $>2$ valgus & not reported & $\begin{array}{c}1.2 \text { valgus( } 6 \\
\text { varus-10 valgus) }\end{array}$ & $1.2(0-5)$ varus & $5(1-8)$ valgus \\
\hline $\begin{array}{c}\text { Type of implant } \\
\text { used }\end{array}$ & $\begin{array}{l}\text { staples(20), ex- } \\
\text { fix(2), screws(2) }\end{array}$ & blade plate & semitubular plate & blade plate & blade plate & $\begin{array}{l}\text { Puddu non- } \\
\text { locking plate }\end{array}$ \\
\hline Time to union & not reported & not reported & not reported & 4.7(3-9) months & not reported & not reported \\
\hline Immobilisation & not reported & 2 weeks & none & hinged brace & 2 weeks & none \\
\hline Weight bearing & not reported & after 6 weeks & immediate & after 6 weeks & at 6-8 weeks & immediate partial \\
\hline $\begin{array}{l}10 \text { year cumulative } \\
\text { survivorship }\end{array}$ & not reported & $64 \%(48-80)$ & not reported & $87 \%(69-100)$ & $82 \%(75-89)$ & not reported \\
\hline $\begin{array}{l}\text { Converted to TKR } \\
\text { or awaiting TKR } \\
\text { at latest follow up }\end{array}$ & $3 / 24$ & $7 / 21$ & none & $3 / 30$ & $12 / 38$ & $2 / 12$ \\
\hline $\begin{array}{l}\text { Mean pre-op HSS } \\
\text { score }\end{array}$ & $58(27-82)$ & not recorded & $65(56-70)$ & $46(20-63)$ & not recorded & 58 \\
\hline $\begin{array}{l}\text { Mean post-op HSS } \\
\text { score }\end{array}$ & $78(40-94)$ & not recorded & $84(61-100)$ & $88(65-99)$ & not recorded & 72 \\
\hline
\end{tabular}

94). Cumulative survival was not presented, but at a mean follow up of 8.3 years, $13 \%$ of knees $(n=3)$ had undergone TKA [8]. Overall this series satisfied 9/16 of Rangel's criteria.

Finkelstein, et al. [5], reported a series of 23 patients who underwent closing wedge DFVO for osteoarthritis of the knee. The surgical technique used was the same as that described by McDermott, et al. [6]. The primary outcome measure used in this study was an un-referenced scoring system. It was therefore not possible to compare outcome against other studies or determine a clinically relevant difference. The 10 year survival assessed by Kaplan-Meier analysis was $64 \%$ (95\% CI $48 \%$ to $80 \%$ ) [5]. Overall this series satisfied 11/16 of Rangel's criteria.

Stahelin, et al. [15] reported the outcome of closing wedge DFVO in 19 patients (21 knees). The indications for surgery included symptomatic mild to moderate lateral compartment osteoarthritis with a valgus deformity of less than 20 degrees. Contra-indications included degenerative change in medial and patellofemoral compartments. Preoperative planning was clearly described with an intended correction of the tibiofemoral angle to 1 to 3 degrees of varus. The surgical technique consisted of a medial closing wedge osteotomy secured by a semi-tubular plate. The authors used an oblique osteotomy to allow circumferential cortical contact of the osteotomy surfaces whereas a horizontal osteotomy would result in a smaller proximal cortical diameter, which could potentially subside into the larger metaphyseal segment. Postoperatively patients were immediately partially weight bearing with crutches for 8 weeks. They were subsequently allowed to fully weight bear if they were pain free even if radiographs showed no callus formation. The mean HSS score improved from 65 (range 56 to 70 ) to 84 (61 to 100) at a mean of five years (range two to twelve years) postoperatively. A statistical analysis was not presented. No patients required conversion to arthroplasty. In eighteen knees (missing data $3 / 21$ patients) the mean difference between the planned correction and the final correction achieved after healing was 1.7 degrees (range 0 to 
Table 3. Table to Show the Number of Complications Reported by Authors of Each Study

\begin{tabular}{|c|c|c|c|c|c|c|}
\hline & Edgerton & Finkelstein & Stahelin & Wang & Backstein & Das \\
\hline Infection & 1 pin tract & 1 (superficial) & 1 (superficial) & & & \\
\hline Haematoma not requiring treatment & & & 2 & & & \\
\hline Pulmonary embolism & & 1 & & & & \\
\hline Deep vein thrombosis & & & 1 & & & \\
\hline Delayed union & 5 & & & & & 1 \\
\hline Non union requiring surgery & 2 & & & 1 & & \\
\hline Peri-prosthetic fracture & & 1 & & & & \\
\hline Removal of metalwork & & & & 13 & & 7 \\
\hline Loss of fixation requiring revision & 4 & 1 & 1 & & & \\
\hline Loss of fixation not requiring revision & 4 & & & & & \\
\hline Stiffness requiring manipulation & & 1 & & & & \\
\hline Total number of complications & 16 & 5 & 5 & 14 & & 8 \\
\hline
\end{tabular}

4 degrees) [15]. Overall the study satisfied 12/16 of Rangel's criteria.

Wang and Hsu [12], reported a series of 30 patients (30 knees) who underwent closing wedge DFVO with a mean follow up of 99 months (range 61 to 169 months). Indications for surgery were non-inflammatory arthritis of the lateral compartment in association with a valgus knee greater than 12 degrees. Exclusion criteria included severe degeneration in other compartments. The surgical technique used was that previously described by McDermott et al. [6]. Postoperatively, patients were immobilised in a hinged knee brace until healing of the osteotomy occurred. Non- weight bearing was maintained until initial healing of the osteotomy was confirmed. Full weight bearing was only allowed at 3 months if good healing was noted. The mean HSS score improved from 46 (range 20 to 63) to 88 (range 65 to 99) at the most recent follow up $(\mathrm{p}<0.001)$. Three patients required conversion to TKA. All 3 had lateral compartment involvement only, and had been corrected to 0 or 1 degree valgus. Cumulative 10 -year survival was $87 \%$ (95\% CI $69 \%$ to $100 \%$ ) [12]. Overall the study satisfied $11 / 16$ of Rangel's criteria.

Das, et al. [16], reported the only series of opening wedge DFVO that was identified by the literature search. Thirteen patients underwent surgery for mild to moderate lateral osteoarthritis associated with genu valgum greater than 10 degrees. Contraindications for surgery included severe medial and patellofemoral degeneration. Following lateral approach to the distal femur, a guide wire was inserted to a hinge point $1 \mathrm{~cm}$ superior to the lateral epicondyle. The osteotomy was created with an oscillating saw ending approximately $5 \mathrm{~mm}$ lateral to the medial cortex. The osteotomy plane was then opened to approximately 3 degrees of valgus. The Puddu plate (Arthrex, Naples, FL,
USA), which is designed with varying spacer-blocks, was then placed over the osteotomy gap and secured proximally with traditional bi-cortical screws and distally with partially threaded cancellous screws. The wedge was filled with allograft. Patients were mobilized partially weight bearing with 2 crutches and a splint for 8 weeks postoperatively or until radiological signs of healing were seen. The mean preoperative HSS score was 58 but no range was provided. The mean HSS improved at both 34 and 74 months postoperatively (HSS score 67 and 72 respectively). The postoperative anatomical axis was corrected to 5 (1-8) degrees valgus. A cumulative survival analysis was not presented but 2 patients required conversion to arthroplasty at 37 and 42 months post-osteotomy. The authors report these patients to have had the most severe pre-operative lateral arthritis and stage II and I medial wear respectively. They felt that DFVO had failed because of advanced wear on the medial side. They also concluded that patients with advanced medial wear had less functional improvement and a lower Lysholm score at follow up. Unfortunately, the data was not clearly reported and no statistical analysis was presented. In addition, no specific comment was made regarding whether any loss of fixation or collapse of osteotomies were noted. This is an important omission as these are both concerns associated with the stability of opening wedge osteotomy when compared to a closing wedge technique.

\section{DISCUSSION}

This systematic review has demonstrated a paucity of high level literature pertaining to DFVO in the treatment of lateral gonarthrosis of the knee. All of the included studies, which met the inclusion/exclusion criteria, reported that good results are achievable with DFVO. However, comparison between them was difficult because there was no 
single outcome measure that was universally used. The HSS score was reported by 4 of the studies $[8,12,15,16]$. The mean pre-operative values ranged from 46 to 65 and these improved at latest follow up to means of between 72 and 88 . Although the populations are not directly comparable, it is interesting to note that recently published figures for lateral UKA report similar HSS values (88 (range 71-95) at 1260 months post-operatively) [18]. However, the results of the included studies do not provide the reader with information about return to sport and impact activities. This is something that is often quoted as an advantage of osteotomy over arthroplasty but no evidence to support this was identified [1].

This review also aimed to evaluate the published literature to determine survivorship of DFVO, with conversion to arthroplasty identified as an endpoint. Three studies reported long term cumulative survival for closing wedge osteotomy series. This ranged from 64 to $82 \%$ at 10 years, and $45 \%$ at 15 years. The single opening wedge series reviewed did not report cumulative survival but did indicate that 2 patients $(17 \%)$ had undergone TKA at the latest follow up (mean 74 months) [16]. However, despite these encouraging figures the literature search failed to identify any controlled trials that could potentially evaluate whether DFVO is associated with delay or avoidance of arthroplasty.

The outcome of DFVO was also assessed by the accuracy of final correction of deformity with respect to the intended degree of correction. The opening wedge technique allows fine-tuning of deformity correction by application of an opening device such as a laminar spreader until the desired angle is achieved. In contrast, in closing wedge osteotomy, the surgeon is very reliant on the accuracy of bony resection.

73 not relevant and excluded
Precise resection of a wedge is technically difficult. It is therefore appropriate to compare the final correction achieved by both techniques with respect to the intended correction. All authors aimed to achieve a final tibiofemoral angle of 0 degrees (i.e. 6 degrees of mechanical varus) except Stahelin, et al. [15] who aimed for a tibiofemoral angle of 1 to 3 degrees of varus and Das et al. [16] who aimed for 3 degrees of valgus. The final tibiofemoral angles achieved by all authors varied between 18 degrees varus to 16 degrees valgus. If the series reported by Edgerton, et al. [8] is excluded then the range of final tibiofemoral angles achieved decreases ( 6 degrees varus to 10 degrees valgus). It is reasonable to exclude this series as the authors used a staple fixation device that they have now abandoned due to a high rate of loss of fixation. Despite this, it remains disappointing to report such a large range of final tibiofemoral angles. The broad range suggests that either accurate correction is not always achieved or that there is some loss of fixation or collapse of osteotomies postoperatively.

Stahelin, et al. [15] suggested that by performing an oblique rather than transverse cortical osteotomy, contact could be maintained throughout the diameter of both proximal and distal fragments thus improving stability. Certainly, the range of final tibiofemoral angles reported by Stahelin, et al. was low, with a final mean tibiofemoral angle within 1.7 degrees (range $0-4$ ) of the intended correction to a tibiofemoral angle of 1 to 3 degrees of varus. In the only opening wedge series [16], the range of final tibiofemoral angles was 1-8 degrees valgus and this is a much smaller range than in some of the closing wedge series (Edgerton $e t$ al. [8] $18^{\circ}$ varus to $16^{\circ}$ valgus, Wang et al. [12] $6^{\circ}$ varus to $10^{\circ}$ valgus). Although it was not necessarily surprising to

\section{PubMed/Medline database search}

(82 studies identified)

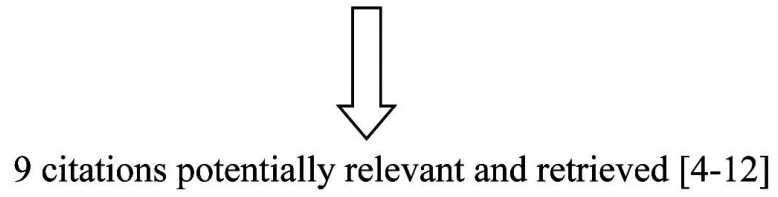

9 citations potentially relevant and retrieved [4-12]

\section{5 excluded}

use of external rather than internal fixation (1) [11]

inclusion of patients with other diagnoses $(2)[9,10]$

use of multiple surgical techniques within one study (1) [17]

duplicate (and shorter) follow up of same patients as an included study (1) $[5,6]$

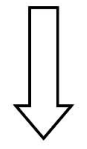

\section{Search of references of all citations retrieved (3 studies identified)}

1 excluded

use of plaster cast rather than internal fixation(1) [14]

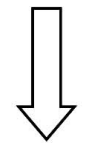

Total of 6 included studies $[4,5,8,12,15,16]$

Fig. (1). Flow of studies. 
see a trend towards a wider range of final angles with the closing wedge technique, the advent of accurate cutting guides which have only recently become available means that this topic needs to be re-visited by prospective study using modern implants and instrumentation.

This study also aimed to determine the complication profile of DFVO (Table 3). In the series reported by Wang, et al. [12] there was a $43 \%$ (13/30) re-operation rate for removal of metalwork. The highest rate of reported complications was $65 \%$ in the study by Edgerton, et al. [8]. This series contained a high number of patients that required re-operation for non-union or loss of fixation. The authors felt that this was due to the method of fixation used and have now abandoned this technique. If the series reported by Backstein [4] (complications not reported) and Edgerton [8] are excluded (in recognition of the fact that this implant is no longer used), then $5.8 \%(5 / 86)$ of osteotomies included in this systematic review required unanticipated re-operation (i.e. excluding removal of metalwork) due to a complication (fracture $(n=1)$, non-union $(n=1)$, stiffness requiring manipulation under anaesthesia $(n=1)$, loss of fixation $(n=2)$ ) and $6.9 \%(6 / 86)$ experienced a complication that did not require further surgery (superficial wound infection $(n=2)$, haematoma $(n=2)$, pulmonary embolism $(n=1)$ and deep vein thrombosis $(\mathrm{n}=1))$.

Complication rates following DFVO may also be influenced by the rehabilitation regimen used and early loading may increase the risk of loss of fixation. The rehabilitation protocols used differed between studies and included immobilisation with a cylinder cast or brace $[4,5$, 12], immediate partial weight bearing for between 6-12 weeks [15] and non-weight bearing for 6 weeks [12]. Potentially relevant parameters such as obesity, smoking and compliance with rehabilitation were not reported. The considerable heterogeneity between series precluded any statistical analysis.

The outcomes of DFVO may be improved by careful selection of patients. This review aimed to establish precise indications for DFVO based on the inclusion and exclusion criteria of the studies reviewed. Unfortunately, these criteria were often poorly reported. This review was unable to determine the optimum timing for DFVO with respect to early or late arthritis because the majority of authors did not report the degree of change required in the lateral compartment for which they considered DFVO to be indicated. Only Edgerton, et al. [8] gave this information and they considered DFVO to be indicated in patients with mild to severe degenerative change.

There was also a lack of consensus regarding whether degenerative change in other compartments was acceptable pre-operatively. Stahelin, et al. [15] excluded patients with disease in other compartments, whereas others did not see this as a contraindication and included them [5, 8, 12]. Edgerton et al. included those with isolated lateral and mild, moderate and severe degenerative change in both the medial and patellofemoral joints and subsequently reported satisfactory results in $86 \%, 80 \%, 30 \%$ and $50 \%$ respectively. However, no statistically significant difference was demonstrated [8]. Wang et al. also included patients with severe patellofemoral and moderate medial compartment wear, but they reported satisfactory results in these patients [12].

Other areas of ambiguity include the importance of preoperative deformity in patient selection. All authors agreed that a valgus deformity was a pre-requisite but only Wang, et al. [12] (greater than 12 degrees valgus) and Stahelin, et al. [15] (less than 20 degrees valgus) described the degree of deformity that they considered to be an indication for DFVO. However, a reason for choosing these values was not stated.

Similarly, there was no consensus between authors with respect to the eligibility of patients for DFVO with respect to age. The range was 20 to 77 years and none of the authors stated that they had excluded patients on the basis of age.

Although a detailed analysis is beyond the scope of this paper, a review of this topic would be incomplete without a comment regarding outcomes of TKA following a failed DFVO. Nelson, et al. [19] reported that not only was arthroplasty more difficult to perform following DFVO but also less successful because the deformity resulting from DFVO was extra-articular and therefore intra-articular correction during TKA could result in ligamentous instability requiring the use of a constrained prosthesis. They also cautioned that the deformity created by DFVO results in an intersection of the lateral femoral condyle by the femoral anatomical axis, which increases the risk of placing the femoral component in less than the desired amount of valgus. Other authors, however, have reported no technical difficulty with conversion to TKA and have reported good functional results $[5,20]$.

The major limitations of this review were the quality of the included studies and their heterogeneity, which prevented any statistical analysis of the available data. This lack of high quality studies reflects the infrequency with which DFVO is performed and the long-term follow-up that is required to assess clinically relevant outcomes. A major concern was that none of the included studies clearly defined the population from which patients had been recruited thus indicating considerable potential for selection bias and also failing in providing the reader with clear indications for DFVO particularly with respect to the degree of arthritis in the lateral and other compartments. In addition, there were few attempts to blind investigators or to ensure accuracy of data collection suggesting that these studies would also have been susceptible to measurement and investigators bias.

\section{CONCLUSIONS}

DFVO offers a potential option in delaying joint replacement surgery but it is technically demanding and requires significant down time for the patient. The included studies report long-term survivorship and good function so clearly there is a role for osteotomy. However, the precise indications for DFVO remain undefined. Some surgeons may consider osteotomy patients and arthroplasty patients as two distinct populations but this is not reflected in the available evidence and the reported series contain young patients with early isolated wear, elderly patients, and those with advanced patella-femoral and medial wear. Further study is therefore required to determine precise indications.

Other areas for further study include a comparison of the outcomes of osteotomy against arthroplasty. This is 
particularly important because this review has shown that there is little evidence to support the popular notion that osteotomy gives the advantages over arthroplasty of allowing high functional loading, and a return to sport and impact activity. In addition, reports of difficulty in performing eventual arthroplasty must necessitate that patients and surgeons have appropriate expectations of outcome from osteotomy and that of subsequent arthroplasty if it is required.

\section{ACKNOWLEDGEMENT}

None declared.

\section{CONFLICT OF INTEREST}

The author(s) confirm that this article content has no conflicts of interest.

\section{REFERENCES}

Santaguida PL, Hawker GA, Hudak PL, et al. Patient characteristics affecting the prognosis of total hip and knee joint arthroplasty: a systematic review. Can J Surg 2008; 51(6): 428-36.

[2] Hanssen AD, Stuart MJ, Scott RD, Scuderi GR. Surgical options for the middle-aged patient with osteoarthritis of the knee joint. J Bone Joint Surg 2000; 82-A(12): 1768-81.

[3] Healy WL, Riley LH. High tibial valgus osteotomy. Clinical review. Clin Orthop 1986; 114: 223-34

[4] Backstein D, Morag G, Hanna S, Safir O, Gross A. Long-term follow-up of distal femoral varus osteotomy of the knee. J Arthroplasty 2007; 22(4 Suppl. 1): 2-6.

[5] Finkelstein JA, Gross AE, Davis A. Varus osteotomy of the distal part of the femur. A survivorship analysis. J Bone Joint Surg Am 1996; 78(9): 1348-52.

[6] McDermott AG, Finklestein JA, Farine I, Boynton EL, MacIntosh DL, Gross A. Distal femoral varus osteotomy for valgus deformity of the knee. J Bone Joint Surg Am 1988; 70-A(1): 110-6.
[7] Aglietti P, Menchetti PP. Distal femoral varus osteotomy in the valgus osteoarthritic knee. Am J Knee Surg 2000; 13(2): 89-95.

[8] Edgerton BC, Mariani EM, Morrey BF. Distal femoral varus osteotomy for painful genu valgum. A five-to-11-year follow-up study. Clin Orthop Relat Res 1993; (288): 263-9.

[9] Terry GC, Cimino PM. Distal femoral osteotomy for valgus deformity of the knee. Orthopedics 1992; 15(11): 1283-9.

[10] Healy WL, Anglen JO, Wasilewski SA, Krackow KA. Distal femoral varus osteotomy. J Bone Joint Surg 1988; 70-A(1): 102-9.

[11] Gugenheim JJ, Brinker Mr. Bone realignment with use of temporary external fixation for distal femoral valgus and varus deformities. J Bone Joint Surg 2003; 85-A(7): 1229-37

[12] Wang JW, Hsu CC. Distal femoral varus osteotomy for osteoarthritis of the knee. J Bone Joint Surg 2005; 87-A(1): 127-33.

[13] Brouwer RW, van Raaij TM, Bierma-Zeinstra SMA, Verhagen AP, Jakma TT, Verhaar JAN. Osteotomy for treating knee osteoarthritis. Cochrane Database of Syst Rev 2007; issue 3. Art. No.: CD004019. DOI: 10.1002/14651858.CD004019.pub3.

[14] Mathews J, Cobb AG, Richardson S, Bentley G. Distal femoral osteotomy for lateral compartment osteoarthritis of the knee. Orthopedics 1999; 21(4): 437-40.

[15] Stahelin T, Hardegger F, Ward JC. Supraconylar osteotomy of the femur with use of compression. Osteosynthesis with a malleable implant. J Bone Joint Surg Am 2000; 82(5): 712-22.

[16] Das D, Sijbesma T, Hoekstra H, M van Leeuwen W. Distal femoral opening-wedge osteotomy for lateral compartment osteoarthritis of the knee. Open Access Surg 2008; 1: 25-9.

[17] Rangel S, Kelsey J, Henry MCW, Moss RL. Critical analysis of clinical research reporting in pediatric surgry: Justifying the need for a new standard. J Pediatr Surg 2003; 38(12): 1739-43.

[18] Volpi P, Marinoni L, Bait C, Galli M, Denti M. Lateral unicompartmental knee arthroplasty: indications, technique and short-medium term results. Knee Surg Sports Traumatol Arthrosc 2007; 15: 1028-34.

[19] Nelson CL, Saleh KJ, Kassim RA, et al. Total knee arthroplasty after varus osteotomy of the distal part of the femur. J Bone Joint Surg Am 2003; 85-A(6): 1062-5.

[20] Cameron HU, Park YS. Total knee replacement after supracondylar femoral osteotomy. Am J Knee Surg 1997; 10: 70. 